\title{
Temporal coherence of individual turbulent patterns in atmospheric seeing
}

\author{
Brian Kern, Ted A. Laurence, Chris Martin, and Paul E. Dimotakis
}

\begin{abstract}
We used a variation of the generalized scidar (scintillation detection and ranging) technique to examine the temporal coherence of turbulent patterns at different altitudes in the atmosphere above Palomar Observatory. This enables us to test the validity of a frozen turbulence hypothesis in the local reference frame of the moving atmosphere. The data set analyzed here contains three turbulent patterns, each at a different altitude, which remain internally coherent over time scales of $0.28-0.41 \mathrm{~s}$. This measurement is significant, because it is made on a $5-\mathrm{m}$ aperture, allowing moving patterns to be tracked over time scales longer than their own lifetimes. (C) 2000 Optical Society of America

OCIS codes: $\quad 010.1080,280.0280,010.1330,010.7060,010.7350,280.7060$.
\end{abstract}

\section{Introduction}

The limitation on the angular resolution of large astronomical telescopes is set by atmospheric seeing, caused by turbulent mixing of volumes of air with different indices of refraction. Adaptive optics systems designed for astronomical applications measure the instantaneous phase aberrations of a reference wave front and apply a real-time phase correction in the optical path. To freeze the instantaneous phase aberrations, however, exposure times must be kept short; $t_{\text {exp }}<t_{\text {blur }}=d / v$, where $t_{\text {blur }}$ is the time it takes a feature of size $d$ to become blurred and $v$ is the bulk wind velocity. For 1-arc sec seeing in visible light $(\lambda \sim 0.5 \mu \mathrm{m})$ the appropriate distance $d$ is Fried's coherence length ${ }^{1} r_{0} \sim 10 \mathrm{~cm}, v \sim 10 \mathrm{~m} / \mathrm{s}, t_{\text {blur }} \sim 10$ ms. This maximum time scale sets stringent limits on the brightness of reference stars used to measure the wave-front phase aberrations.

Although it is necessary to observe aberrations on time scales of $10 \mathrm{~ms}$ to avoid blurring, the aberrations in two exposures $10 \mathrm{~ms}$ apart may be highly correlated. A limiting case of this correlation can be traced back to Taylor's frozen turbulence hypothesis. ${ }^{2}$ In the limiting form of the hypothesis turbulent patterns are effectively frozen on the time scales re-

The authors are with the California Institute of Technology, MS 405-47, 1201 East California Boulevard, Pasadena, California 91125. The e-mail address of B. Kern is bdk@srl.caltech.edu.

Received 28 September 1999; revised manuscript received 31 May 2000.

0003-6935/00/274879-07\$15.00/0

(C) 2000 Optical Society of America quired for the bulk wind to carry the patterns across the telescope pupil. A maximum observable coherence time can be defined, $t_{\max }=D / v$, where $D$ is the telescope diameter. For $D=5 \mathrm{~m}, t_{\max } \sim 0.5 \mathrm{~s}$, or $t_{\text {max }} \sim 50 t_{\text {blur }}$.

If this maximum coherence can be exploited, then measurements of the wave-front phase aberrations can have an effective exposure time as large as $t_{\text {eff }} \sim$ $50 t_{\text {blur. }}$ Use of this extra information can be called wave-front prediction, in which knowledge of past wave-front measurements can be used to augment an instantaneous wave-front measurement. ${ }^{-5}$ Long coherence time scales also allow one to expand the isoplanatic patch, ${ }^{6}$ from $\theta_{\text {iso }}=r_{0} / h$ to $\theta_{\text {iso, } \|}=D / h$ parallel to the wind.

For adaptive optics systems, coherence time enhancements translate into less-stringent requirements on the brightness and proximity of natural guide stars, which in turn correspond to an increase in sky coverage. At its maximum the increase in effective exposure time allows for the use of guide stars fainter by a factor of $\sqrt{50}$, which in turn yields an increase in the number of usable guide stars by a factor of $(\sqrt{50})^{3 / 2}$. When combined with the expanded isoplanatic patch, use of the maximum coherence time would yield an increase in sky coverage by a factor of $50^{7 / 4} \approx 1000$ for a 5 -m telescope, under the conditions described above. In this paper we describe a measurement of this coherence time; we measure it to be approximately 20-30 times $t_{\text {blur }}$, corresponding to a potential sky coverage increase by a factor of 200-400.

The method we describe to determine the coherence of turbulent patterns is a variation on the gen- 
eralized scidar (scintillation detection and ranging) technique. The scidar technique was developed by Vernin and co-workers ${ }^{7,8}$ and later generalized ${ }^{9-12}$ to examine ground-layer turbulence. The scidar technique involves examining the cross correlation of scintillation patterns cast by binary-star pairs. Assuming that the effect of the turbulence is described by Kolmogorov models, ${ }^{13}$ the distribution of $C_{n}{ }^{2}$ versus height can be estimated from the cross correlation, with a sensitivity that increases with height. The generalized scidar technique moves the observation plane (conjugate to the detector) away from the telescope pupil so that the effects of ground-layer turbulence, to which one would otherwise be insensitive, can be imaged. Since the detector is no longer conjugate to the pupil plane, the projections of the telescope aperture from the two stars do not coincide. As we chose more-distant binary pairs, or moredistant observation planes, the illumination overlap decreases (see Ref. 14). The variation we employ on this technique is to use more-distant binary pairs and move the observation plane far enough that the two illumination patterns no longer overlap. This yields a better vertical resolution in the resulting analysis and makes it possible to use the autocorrelation of a single star as well as the cross correlation between the two stars.

\section{Observations}

On the evening of 18 March 1996 we placed a Sony Model XC-75 monochrome video camera behind the $F / 16$ Cassegrain focus of the Palomar Observatory 5 -m telescope. The video output, at 30 frames/s, was captured on a Sony Hi8 VCR then later played back and digitized to produce monochrome 8-bit, $(640 \times 480)$-pixel images. There were no filters used in these observations, so the camera was sensitive to light between 400 and $800 \mathrm{~nm}$. The camera lens, with everything in its nominal position, produced an in-focus image with a plate scale of $0.1 \mathrm{arc} \mathrm{sec} / \mathrm{pixel}$. Changing only the telescope focus, we obtained an out-of-focus image, which is equivalent to having the detector conjugate to an observation plane far below the telescope pupil (in focus is infinitely far below the telescope pupil). With this setup we could view the stars in focus to determine the seeing and then easily move to the observation plane below the telescope pupil. This instrumentation is simpler than comparable systems proposed or demonstrated in the past.

The targets of our observations were binary stars, selected so that the two defocused images were well sampled and so that both fit on a CCD frame. The rest of this paper concerns observations of Aitken Double Star (ADS) 11853 A and B, with a separation of 22 arc sec and visual magnitudes 4.6 and 5.0. The 22 -arc sec separation allowed us to move the telescope focal plane longitudinally (out of focus) by 13 $\mathrm{cm}$ before the two stellar illumination patterns overlapped. This distance makes the detector conjugate to an observation plane $50 \mathrm{~km}$ below the telescope pupil, yielding an observation plane scale of $2.5 \mathrm{~cm} /$ pixel. The geometry is illustrated in Fig. 1. Thus

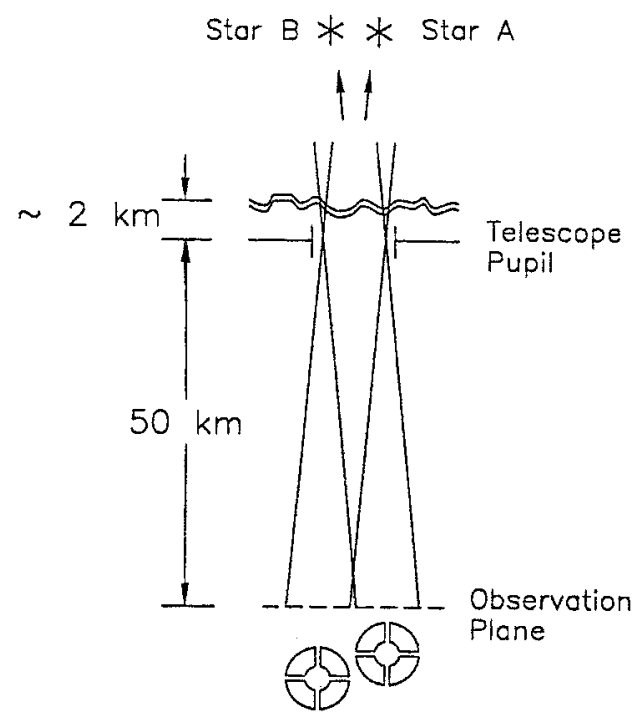

Fig. 1. Schematic observing geometry (not to scale). The marginal rays of both stellar images are shown, and the illumination patterns are shown as they appear in the observation plane, which is conjugate to the detector.

the 500-cm pupil gave two 200-pixel-diameter illumination patterns, with centers separated by 22 arc sec, or 220 pixels. A sample frame, showing the out-offocus images of the two stars, is shown in Fig. 2. The true resolution in the observing plane is set not by the pixel scale but by the size of the first Fresnel zone, which is $\sim \sqrt{z \lambda}=16 \mathrm{~cm}$, or $\sim 6$ pixels. Here $z$ is 50 $\mathrm{km}$, the distance from the observation plane to the pupil plane.

The camera operates at the standard video rate of $30 \mathrm{~Hz}$. After the observing run we digitized 256

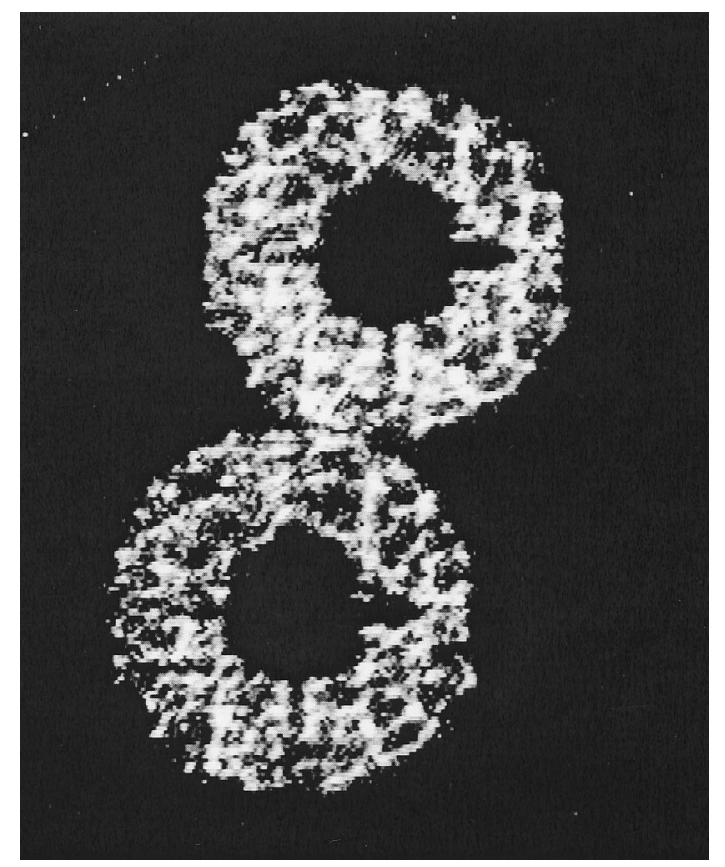

Fig. 2. Sample digitized frame, containing two out-of-focus stellar images. 
consecutive frames, corresponding to $8.5 \mathrm{~s}$. The digitized data were split into two data cubes, each a $(256 \times 256 \times 256)$-element time series of the images of a single star. We refer to the data cubes as data cube A, corresponding to star ADS $11853 \mathrm{~A}$, and data cube B, corresponding to ADS $11853 \mathrm{~B}$.

\section{Analysis}

\section{A. Correlation Functions}

The common use of generalized scidar data is to obtain a plot of $C_{n}{ }^{2}$ versus height. For three reasons we do not do this with our data. First, our measurements cannot confirm the applicability of Kolmogorovderived structure functions, and, in fact, we have reason to believe that some of the effects we see are from non-Kolmogorov motions. Some scintillation structures we see in other data sets seem to be due to convective currents passing through the telescope slit, where there is no reason to believe that the turbulence follows a fully developed Kolmogorov spectrum. This is not a highly significant observation but rather a cautionary statement about our assumptions. Second, if we were to assume a Kolmogorov turbulent distribution, the simplest theories for transformation from amplitude fluctuations to $C_{n_{2}}{ }^{2}$ would be applicable in the weak fluctuation limit $\left(\sigma_{x}{ }^{2}\right.$ $<1$ ), where fluctuations are much smaller than the mean amplitude. Fluctuations grow with propagation distance; with the observation plane located 50 $\mathrm{km}$ from the nearest phase aberrations the fluctuations fall into the strong-fluctuation limit. This means that the transformation from amplitude fluctuations to $C_{n}{ }^{2}$ does not follow the same relationship as is used in generalized scidar. Independent of the other reasons mentioned here, this difference would mean that our estimates of $C_{n}{ }^{2}$ would not be directly comparable with estimates taken from generalized scidar measurements. The third reason our data do not easily yield quantitative turbulent strengths is that our exposure time is longer than $t_{\text {blur. This }}$ causes streaking, in which the instantaneous scintillation pattern is convolved with a line whose length depends on wind speed and exposure time. This results in a truncation of the scintillation power spectrum, which is another complication in transforming amplitude fluctuations to turbulent strength. Although our long exposure time does reduce our sensitivity to high-velocity patterns, it does not affect our measurements of the coherence of an individual pattern as observed by our camera. We would have preferred to use a shorter exposure time so that we would be more sensitive to high-velocity structures, but we obtained our observing time serendipitously, and we were not able to adjust the default exposure time.

With two separate data cubes we have two basic actions available. We can perform an autocorrelation on either data cube independently, or we can perform a cross correlation between the two data cubes. To avoid aliasing effects during use of fast Fourier transforms, for any correlation analysis the data cubes were padded with blank entries so that the data cubes correlated had $512 \times 512 \times 512$ elements each. We performed the correlations on Caltech's Intel Paragon supercomputer, where a $512^{3}$-element double-precision three-dimensional autocorrelation is calculated in $\sim 30 \mathrm{~s}$. The correlations we performed are correlation functions in agreement with the conventional definition, i.e.,

$$
\begin{aligned}
& B_{\chi}(\boldsymbol{\rho}, \tau)=\langle\chi(\mathbf{r}, t) \chi(\mathbf{r}+\boldsymbol{\rho}, t+\tau)\rangle, \\
& C_{\chi}(\boldsymbol{\rho}, \tau)=\left\langle\chi_{\mathrm{A}}(\mathbf{r}, t) \chi_{\mathrm{B}}(\mathbf{r}+\boldsymbol{\rho}, t+\tau)\right\rangle,
\end{aligned}
$$

where $\chi$ is the log amplitude, $B_{\chi}$ is an autocorrelation, $C_{\chi}$ is a cross correlation, and subscripts $\mathrm{A}$ and $\mathrm{B}$ denote stars A and B.

Because of our long exposure times $\left(t_{\exp }=33 \mathrm{~ms}>\right.$ $t_{\text {blur }}$ ), the correlation for a high-velocity pattern is greatly reduced. In a relative sense the measured correlation of zero-velocity turbulence is disproportionately high. When examining other turbulent features, we would like to filter out the zero-velocity turbulence (which we show below is most likely associated with dome turbulence), since the wings of the correlation function from the zero-velocity turbulence obscure the correlation from fast-moving turbulent layers. To remove the zero-velocity turbulence, we apply a simple filter to the temporal frequencies,

$$
F\left(f_{\tau}\right)= \begin{cases}0, & \left|f_{\tau}\right| \leq 1.875 \mathrm{~s}^{-1} \\ 1, & \left|f_{\tau}\right| \geq 1.875 \mathrm{~s}^{-1}\end{cases}
$$

This filter removes features from the correlation functions that change on time scales less than $0.27 \mathrm{~s}$, or eight frames. This specific frequency cutoff was determined empirically as the cutoff that removed the zero-velocity features from the correlation function, as well as removing any static patterns. Since this is a square filter in frequency space, there is ringing in the temporal dimension of correlation space, but the ringing is easily understood and accounted for where it is important. A series of slices through the filtered autocorrelation function are shown in Fig. 3. We call $B_{F}(\boldsymbol{\rho}, \tau)$ the filtered autocorrelation function and $C_{F}(\boldsymbol{\rho}, \tau)$ the filtered cross correlation.

\section{B. Motions of Independent Patterns}

We can use the autocorrelation information to determine wind speed, direction, and evolution of correlation strength of any pattern that remains coherent between frames. ${ }^{15}$ To develop a systematic technique for identifying separate patterns and their speeds, we derive a convective autocorrelation function from $B_{F}(\boldsymbol{\rho}, \tau)$. To examine patterns that are rigidly translated from one frame to the next, we integrate in $(\boldsymbol{\rho}, \tau)$ space along straight lines through $(\boldsymbol{\rho}, \tau)=(0,0)$,

$$
L_{B_{F}}(v, \phi)=\frac{1}{T} \int_{0}^{T} B_{F}[v \cos (\phi) \tau, v \sin (\phi) \tau, \tau] \mathrm{d} \tau .
$$



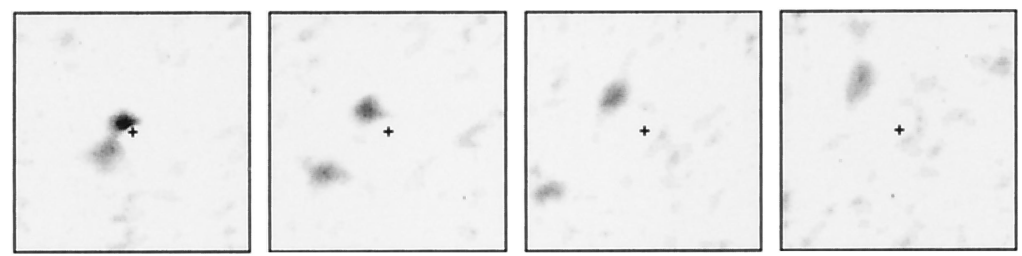

Fig. 3. Slices of $B(\boldsymbol{\rho}, \tau)$ at $\tau=0.07,0.13,0.20$, and $0.27 \mathrm{~s}$. Cross is origin, where zero-velocity peak has been filtered out. Each slice measures $310 \mathrm{~cm} \times 310 \mathrm{~cm}$.

This formulation is substantially similar to the convective autocorrelation function used in Ref. 16, except that we use $(v, \phi)$ instead of $\left(v_{x}, v_{y}\right) . \quad L_{B_{F}}(v, \phi)$ is shown in Fig. 4. Two peaks are clearly visible in the $(v, \phi)$ space, with bulk velocities near 3 and $8 \mathrm{~m} / \mathrm{s}$. Note that the filtering produces a distinct dropoff at low velocities. One would expect that the $F$ filter will remove features that move less than the Fresnel zone size, $16 \mathrm{~cm}$, in $0.27 \mathrm{~s}$, setting a lower detection limit of $0.6 \mathrm{~m} / \mathrm{s}$.

We wish to establish a correlation function for each pattern individually,

$$
B_{F, i}(\tau)=B_{F}\left[v_{i} \cos \left(\phi_{i}\right) \tau, v_{i} \sin \left(\phi_{i}\right) \tau, \tau\right], \quad i=1,2 .
$$

To determine the appropriate values of $v_{i}$ and $\phi_{i}$ for each pattern, we start with an approximate speed and direction as determined by $L_{B_{F}}(v, \phi)$. For each time slice we find the local maximum in $B_{F}(\boldsymbol{\rho}, \tau)$ near the approximate position. We then fit a line to the maximum positions, determining $v_{i}$ and $\phi_{i}$.

To estimate the uncertainty in $B_{F, i}(\tau)$, we let

$$
\begin{aligned}
{\sigma_{B F, i}}^{2}(\tau)= & \frac{1}{7} \sum_{n=1}^{7} B_{F}\left[v_{i} \cos \left(\phi_{i}+n \pi / 4\right) \tau,\right. \\
& \left.v_{i} \sin \left(\phi_{i}+n \pi / 4\right) \tau, \tau\right]^{2}
\end{aligned}
$$

[the temporal filter $F\left(f_{\tau}\right)$ ensures that $\left\langle B_{F}(\boldsymbol{\rho}, \tau)\right\rangle=0$ ]. There is no particular significance in the choice of points to be used in the average. Plots of $B_{F, 1}(\tau)$ and $B_{F, 2}(\tau)$ are shown in Figs. 5 and 6.

\section{Correlation Decay in Moving Patterns}

This study, we believe, is the first to measure an autocorrelation over time scales comparable with

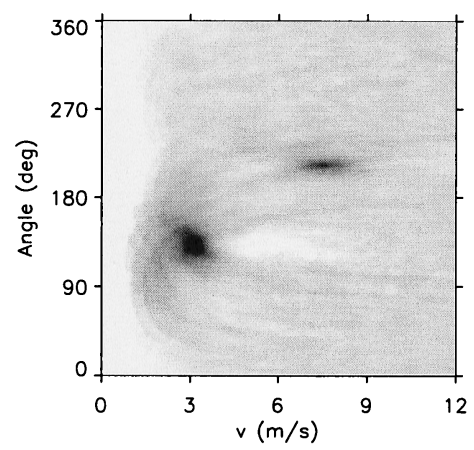

Fig. 4. $L_{B F}(v, \phi)$, the convective autocorrelation function derived from $B_{F}(\boldsymbol{\rho}, \tau)$. $\phi$ measured in degrees $\mathrm{N}$ of $\mathrm{E}$. their decay times. We cannot reliably discriminate between different forms of the decay, i.e., exponential decay, linear decay, and the like. We adopt an exponential fit for these data. We apply a $\chi^{2}$ fit of a decaying exponential, $B_{F, i}^{\text {fit }}(\tau)=a_{i} \exp \left(-\tau / \tau_{\text {decay }, i}\right)$, to each plot. Points where the peak has moved less than the Fresnel zone size away from the zerovelocity (dome turbulence) peak, $v_{i} \tau<\sqrt{z \lambda}$, are ignored. The fits are extended out to where the patterns have moved off the telescope, $v_{i} \tau=D$. In the definition of $B_{\chi}(\boldsymbol{\rho}, \tau)$ [Eq. (1)] an average correlation is used so that the fact that fewer pixels are

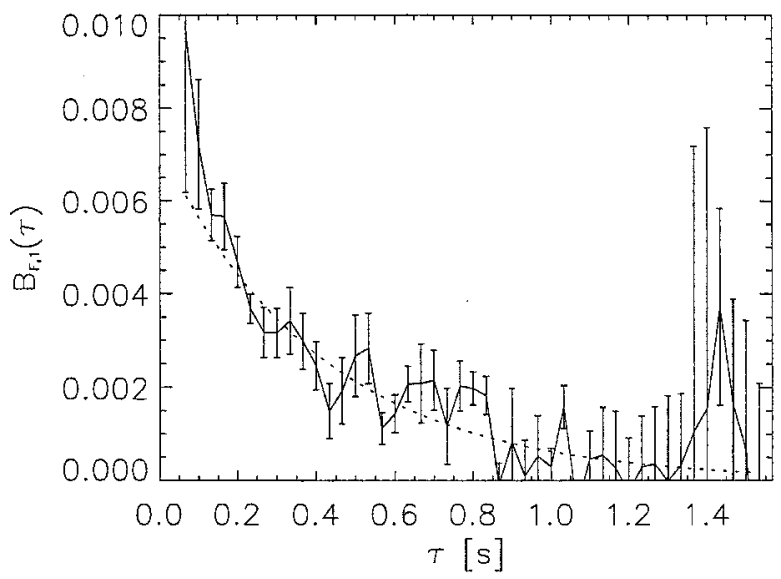

Fig. 5. $B_{F, 1}(\tau)$, filtered correlation function for $3-\mathrm{m} / \mathrm{s}$ pattern. Dotted curve, exponential fit, with $\tau_{\text {decay, } 1}=0.41 \mathrm{~s}$.

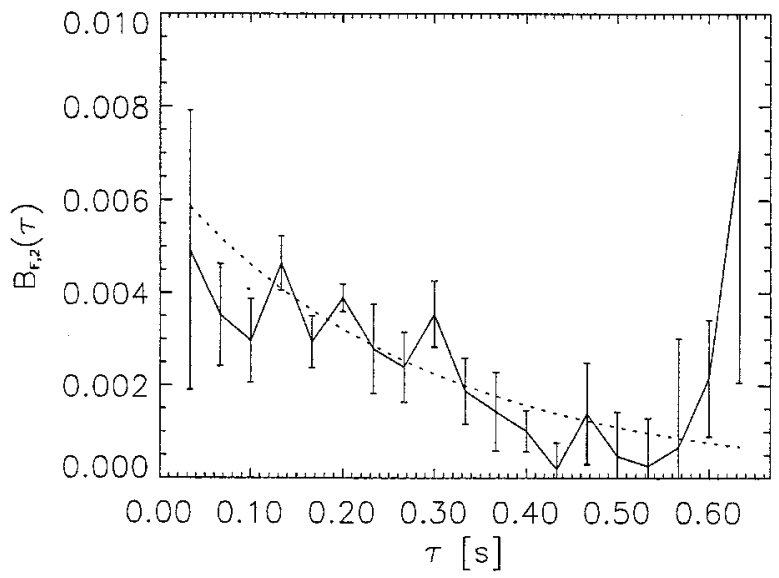

Fig. 6. $B_{F, 2}(\tau)$, filtered correlation function for $8-\mathrm{m} / \mathrm{s}$ pattern. Dotted curve, exponential fit, with $\tau_{\text {decay }, 2}=0.28 \mathrm{~s}$. 
Table 1. Measured Parameters of Turbulent Patterns

\begin{tabular}{cccc}
\hline Speed $(\mathrm{m} / \mathrm{s})$ & Direction $\left({ }^{\circ} \mathrm{N}\right.$ of E) & Decay time $(\mathrm{s})$ & Height $(\mathrm{km})$ \\
\hline 0.0 & - & $0.31 \pm 0.06$ & $0 \pm 0.3$ \\
3.2 & 132 & $0.41 \pm 0.04$ & $1.3 \pm 0.3$ \\
7.8 & 212 & $0.28 \pm 0.05$ & $3.7 \pm 0.3$ \\
\hline
\end{tabular}

involved in the correlation at large $\rho$ does not affect the value of the average correlation but the errors at large $\rho$, or equivalently the errors in $B_{F, i}(\tau)$ for large $\tau$, grow larger. The results of the fit are shown in Table 1. The reduced $\chi^{2}$ of the fits were 1.4 and 1.5 , with 45 and 19 degrees of freedom for the 3.2- and the $7.8-\mathrm{m} / \mathrm{s}$ patterns, respectively. These large values of $\chi^{2}$ imply that the true errors in $B_{F, i}(\tau)$ are larger than estimated by Eq. (6) or that the true form of the decay is not exponential. The fact that both $B_{F, 1}(\tau)$ and $B_{F, 2}(\tau)$ have anomalously large values near the values of maximum $\tau$ is not statistically significant and seems to be coincidental. Reducing the range of $\tau$ values used to fit the exponentials by as much as $30 \%$ does not change the extracted $\tau_{\text {decay }, i}$ by more than the quoted fit errors.

\section{Heights of Moving Patterns}

By using the filtered cross correlation $C_{F}(\boldsymbol{\rho}, \tau)$ in addition to the autocorrelation, we can determine the heights of these layers, in a similar way to the technique used by scidar. Since we have already determined $v_{i}$ and $\phi_{i}$ from $B_{F}(\boldsymbol{\rho}, \tau)$, we can determine a $\rho_{\text {off }, i}$ intercept, which gives us a height from

$$
h_{i}=\left\|\boldsymbol{\rho}_{\text {off, } i}\right\| / \theta,
$$

where $\theta$ is the binary separation angle, 22 arc sec in this case. We find the local maxima in $C_{F}(\boldsymbol{\rho}, \tau)$ near the line defined by $v_{i}$ and $\phi_{i}$ and average their offsets from that line. That average offset, $\rho_{\text {off }, i}$, gives the height of each layer. The heights of the layers are listed in Table 1. As a consistency check the direction of $\boldsymbol{\rho}_{\text {off }, i}$ agrees perfectly with the direction of the binary separation in each case.

\section{E. Dome Turbulence}

To investigate the zero-velocity turbulence, we use the unfiltered autocorrelation, $B_{\chi}(0, \tau)$. We fit a decaying exponential to $B_{\chi}(0, \tau)$ to determine $\tau_{\text {decay }}$. We do not have a simple way to evaluate $\sigma_{B \chi}{ }^{2}(\tau)$ in this case, so we weight each data point equally when fitting. We expect that $B_{\chi}(0,0)$ contains contributions from a number of different turbulent patterns, not all of which need to remain coherent long enough to be detectable to our analysis. We ignore the data points with $\tau<0.07 \mathrm{~s}$ (two frames) when we fit the decay time. We can extend the fit to arbitrarily large values of $\tau$, since a peak with zero velocity never leaves the telescope aperture. However, $B_{\chi}(0, \tau)$ drops below zero when $\tau$ exceeds $\sim 1.5 \mathrm{~s}$, which implies either a significant noise source or effects that go beyond the typical assumption of statistically

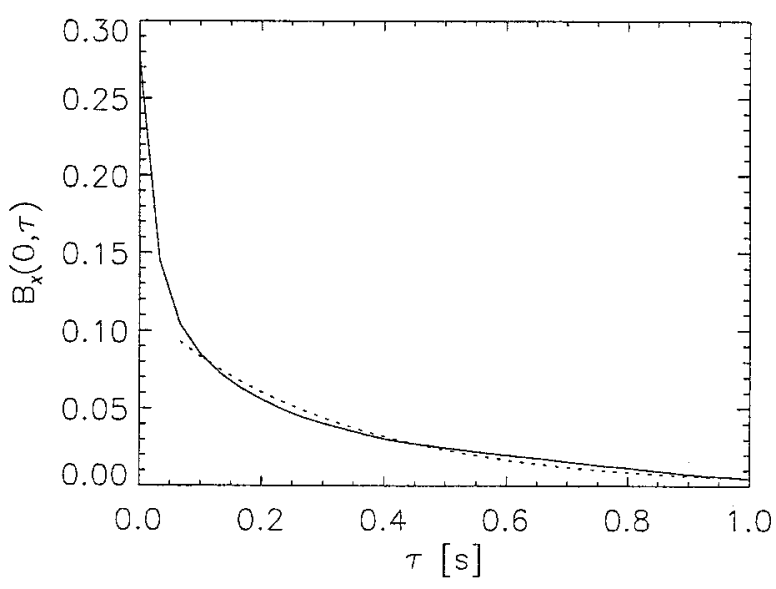

Fig. 7. $B_{\chi}(0, \tau)$, unfiltered correlation function for zero-velocity pattern, with no error estimates. Dotted curve, exponential fit, with $\tau_{\text {decay }, 0}=0.31 \mathrm{~s}$.

independent fluctuation patterns. Assuming that $\tau_{\text {decay, } 0}$ for zero-velocity turbulence is comparable with that for nonzero velocities, $\sim 0.3 \mathrm{~s}$, we extend the zero-velocity fit to $1 \mathrm{~s}, \sim 3 \tau_{\text {decay }, 0}$. Without a simple estimate of $\sigma_{B x}{ }^{2}(\tau)$ it is not clear how well the exponential fit applies to the zero-velocity data. Figure 7 shows that the exponential decay might not be entirely applicable.

We can determine the height of the zero-velocity pattern, using $C_{\chi}(\boldsymbol{\rho}, \tau)$, the unfiltered cross correlation. We find no deviation from $\rho=0$, to an accuracy of $\sim 2.5 \mathrm{~cm}$ ( 1 pixel), or $0.3 \mathrm{~km}$ in height. This height limit leads us to believe that the zero-velocity patterns are dome turbulence and/or slow-moving ground-layer turbulence.

\section{Discussion}

Previous studies have examined the coherence time of turbulence. These studies have been performed in the laboratory ${ }^{17}$ and on astronomical data, ${ }^{15,16,18}$ with both scintillation analysis and Hartmann wave-front sensor data. The laboratory data suggest a decay time of $<1 \mathrm{~ms}$, whereas the astronomical data suggest times of $60 \mathrm{~ms},>200 \mathrm{~ms}$, and $\geq 60$ $\mathrm{ms}$. We do not have enough information to scale the laboratory data properly to compare the decay time with our measurements. The studies that use astronomical data differ from our study in that the longest time scales over which the decay times were observed were smaller than the decay time itself. In part because of our larger telescope aperture, in our study we are able to observe the patterns for $\sim 3 \tau_{\text {decay. }}$. Previous studies also did not publish any error estimates on their coherence time measurements. Of course, it is expected that different observations will yield different coherence times, even observations at different times on the same site with the same instrumentation and with the same reduction methods.

Tatarskii ${ }^{19}$ makes a dimensional argument, on the 
basis of Kolmogorov's assumptions, ${ }^{13}$ for the expected $\tau_{\text {decay }}$,

$$
\tau_{\text {decay }} \sim \frac{l}{v_{l}} \approx \frac{l}{(\epsilon l)^{1 / 3}}=\frac{l^{2 / 3}}{\epsilon^{1 / 3}},
$$

where $l$ is a size scale of interest, $v_{l}$ is the internal velocity at size scale $l$, and $\epsilon$ is the specific energy dissipation rate. A similar dimensional argument yields

$$
\left(\epsilon L_{0}\right)^{1 / 3} \sim v
$$

where $v$ is the bulk wind velocity and $L_{0}$ is the outer scale of the turbulence. Combining these two equations, we get

$$
\tau_{\text {decay }} \sim \frac{\left(l^{2} L_{0}\right)^{1 / 3}}{v} .
$$

The pertinent length scale in our scintillation measurements is the Fresnel zone size, $\sqrt{z \lambda}$. Since the scintillation power spectrum peaks at the Fresnel zone size, most of the coherence effects we see are taking place at this size scale. We also assume that $v_{l}$ for this size scale is comparable with the bulk wind velocity. With $l \approx \sqrt{z \lambda}$ and our measurements of $\tau$ and $v$ (see Table 1 ) we still have no independent measurement of $L_{0}$ to check the validity of this relationship. If we make the gross approximation that $10 \mathrm{~m}<L_{0}<1 \mathrm{~km}$, then we expect $\tau_{\text {decay, } 1} \sim 200-900$ $\mathrm{ms}$ for the $3-\mathrm{m} / \mathrm{s}$ pattern, $\tau_{\text {decay }, 2} \sim 80-375 \mathrm{~ms}$ for the $8-\mathrm{m} / \mathrm{s}$ pattern. If we turn this around and solve for $L_{0}$, then, for the $3-\mathrm{m} / \mathrm{s}$ pattern, $L_{0} \approx 90 \mathrm{~m}$ is consistent, and, for the $8-\mathrm{m} / \mathrm{s}$ pattern, $L_{0} \approx 400 \mathrm{~m}$ is consistent. These numbers are somewhat large but still in the range of previously measured values for $L_{0}$. An enormous range of values of $L_{0}$ have been measured with different methods, ${ }^{11}$ ranging from $2.5 \mathrm{~m}$ to $>2 \mathrm{~km}$. Given this range, our results are broadly consistent with prior measurements, considering that only dimensional arguments were used in our estimate.

The dome turbulence (zero velocity) does not submit itself to a similar analysis, first because the projected bulk velocity is zero (the bulk motion is most likely along the line of sight) and second because, if the turbulence is predominately convective, it need not give results similar to those of fully developed Kolmogorov turbulence.

It is worth mentioning that the use of the terms correlation and decay in this analysis refers only to the spatial correlation of particular flow structures.

The decay of this correlation corresponds to a loss of coherence in a Fourier sense but does not correspond to changes in the actual turbulent properties of the flow. For example, the merging of turbulent vortices to produce a stronger vortex will produce a decay of the spatial correlation in our measurements but does not correspond to a decay in the turbulent properties of the flow.

\section{Conclusions}

We have separated the scintillation patterns from a binary pair into three distinct layers. The three patterns are all tracked for approximately three times their coherent lifetimes, which are $0.28-0.41 \mathrm{~s}$. These numbers are significantly larger than coherence times measured in previous studies. The data for these numbers were taken over a larger aperture than in previous studies, allowing for coherence to be observed for a longer time span, most importantly, for a time span longer than their own lifetimes.

The method demonstrated in this study is simpler than methods used in prior studies. It would be straightforward to set up a routine monitoring program with this technique. All the analysis presented here was extracted from $8 \mathrm{~s}$ worth of data, with identification and examination of separate patterns performed in a nearly automated fashion. A routine monitoring program would allow for determination of the overall distribution of coherence times at each altitude, as well as allow for testing of ideas of wave-front prediction. An additional strength of this method not yet mentioned is that, by use of a cross correlation of stars that do not overlap on the detector, the difference in magnitudes of the two stars does not affect the data quality, to the extent that both stars are bright enough to be well exposed. This translates to a larger number of usable binary pairs than would be practical with generalized scidar, in which the cross correlation of the two stars must be disentangled from the autocorrelation of the two individual stars. Our data quality would improve with the use of a shorter exposure time, reducing the smearing of high-velocity patterns. This would make us sensitive to any higher-velocity structures, if they are present.

We thank Lee Armus for supplying our $8.5 \mathrm{~s}$ of observing time, Dan Lang for technical help, and the Palomar staff for accommodating our nonstandard observing habits. This study was supported by Caltech President's Fund PF-404 and National Science Foundation (NSF) grant AST-9618811.

\section{References}

1. D. L. Fried, "Optical resolution through a randomly inhomogeneous medium for very long and very short exposures," J. Opt. Soc. Am. 56, 1372-1379 (1966).

2. G. Taylor, "The spectrum of turbulence," Proc. R. Soc. London 164, 476-490 (1938).

3. F. Rigaut, G. Rousset, P. Kern, J. C. Fontanella, J. P. Gaffard, F. Merkle, and P. Léna, "Adaptive optics on a $3.6 \mathrm{~m}$ telescope: results and performances," Astron. Astrophys. 250, 280-290 (1991).

4. M. B. Jorgenson and G. J. M. Aitken, "Wavefront prediction for adaptive optics," in Proceedings of the ESO Satellite Conference on Active and Adaptive Optics, F. Merkle, ed. (European Southern Observatory, Garching, Germany, 1993), Vol. 48, pp. 143-148.

5. C. Schwartz, G. Baum, and E. N. Ribak, "Turbulence-degraded wave fronts as fractal surfaces," J. Opt. Soc. Am. A 11, 444451 (1994). 
6. P. Léna, "Astrophysics with adaptive optics: results and challenges," in Adaptive Optics for Astronomy D. Alloin and J. M. Mariotti, eds. (Kluwer Academic, Boston, Mass., 1994), pp. 321-332.

7. J. Vernin and F. Roddier, "Experimental determination of twodimensional spatiotemporal power spectra of stellar light scintillation. Evidence for a multilayer structure of the air turbulence in the upper troposphere," J. Opt. Soc. Am. 63, 270-273 (1973).

8. A. Rocca, F. Roddier, and J. Vernin, "Detection of atmospheric turbulent layers by spatiotemporal and spatioangular correlation measurements of stellar-light scintillation," J. Opt. Soc. Am. 64, 1000-1004 (1974).

9. A. Fuchs, M. Tallon, and J. Vernin, "Folding-up of the vertical atmospheric turbulence profile using an optical technique of movable observing plane," in Atmospheric Propagation and Remote Sensing III, W. A. Flood and W. B. Miller, eds., Proc. SPIE 2222, 682-692 (1994).

10. V. A. Klückers, N. J. Wooder, M. A. Adcock, T. W. Nicholls, and J. C. Dainty, "Results from SCIDAR experiments," in Image Propagation through the Atmosphere, C. Dainty and L. R. Bissonnette, eds., Proc. SPIE 2828, 234-243 (1996).

11. R. Avila, J. Vernin, and E. Masciadri, "Whole atmosphericturbulence profiling with generalized scidar," Appl. Opt. 36, 7898-7905 (1997).

12. A. Fuchs, M. Tallon, and J. Vernin, "Focusing on a turbulent layer: principle of the 'generalized SCIDAR," Publ. Astron. Soc. Pac. 110, 86-91 (1998).

13. A. N. Kolmogorov, "Dissipation of energy in locally isotropic turbulence," Dokl. Akad. Nauk SSSR 32, 16-18 (1941).

14. V. A. Klückers, N. J. Wooder, T. W. Nicholls, M. J. Adcock, I. Munro, and J. C. Dainty, "Profiling of atmospheric turbulence strength and velocity using a generalised SCIDAR technique," Astron. Astrophys. Suppl. Ser. 130, 141-155 (1998).

15. J. L. Caccia, M. Azouit, and J. Vernin, "Wind and $C_{N}^{2}$ profiling by single-star scintillation analysis," Appl. Opt. 26, 1288-1294 (1987).

16. M. Schöck and E. J. Spillar, "Measuring wind speeds and turbulence with a wave-front sensor," Opt. Lett. 23, 150-152 (1998).

17. P. J. Gardner, M. C. Roggemann, and B. M. Welsh, "Quantification of frozen flow properties for a turbulent mixing layer of helium and nitrogen gas," in Image Propagation through the Atmosphere, C. Dainty and L. R. Bissonnette, eds., Proc. SPIE 2828, 256-265 (1996).

18. E. Gendron and P. Léna, "Single layer atmospheric turbulence demonstrated by adaptive optics observations," Astrophys. Space Sci. 239, 221-228 (1996).

19. V. I. Tatarskii, The Effects of the Turbulent Atmosphere on Wave Propagation (Israel Program for Scientific Translations, Jerusalem, 1971). 